\title{
An Analytical Method for Optimum Frequency Domain Design in Control Applications
}

\author{
Gary L. Dempsey, Eugene S. McVey \\ Bradley University/University of Virginia
}

\begin{abstract}
Our paper will revisit a method that was first introduced at Yale University in the late 1950s by Bower and Schultheiss in their linear control textbook, Introduction to the Design of Servomechanisms. Control system design normally requires several design iterations in a simulation environment to meet a set of system specifications. The analytical method discussed here comprises the first design iteration but normally results in a design that is close to optimum, i.e., the resulting system will have maximum stability margin and bandwidth. The method eliminates trial and error procedures, can accommodate time and frequency domain specifications, and can be applied to continuous-time or sampled-data systems.
\end{abstract}

\section{Introduction}

Undergraduate control theory textbooks and courses have typically been heavily weighted to control system analysis while design approaches have received less attention. Design approaches are many times reduced to cookbook methods that are not optimum for a given application. The cookbook methods and lack of design examples do not provide insight into how to modify the controller if a set of system specifications is not met. Therefore, students are unable to solve problems they have not seen in the course or textbook or rely on trial and error procedures to meet specifications. Obviously, analytical design methods for the controllers would be beneficial to undergraduate engineering students and to the control theory community.

Our paper will revisit a method that was first introduced at Yale University in the late 1950s by Bower and Schultheiss ${ }^{1}$. The method was illustrated for several minimum-phase continuous-time systems and a system modeled as first-order with time delay. During this period Dr. J. E. Gibson taught the method in control theory courses at Purdue University. The method was observed later in other textbooks although not in its entirety ${ }^{2-4}$. The authors have presented the method in their control theory courses at Bradley University, Purdue University and the University of Virginia.

The paper is divided into the following five sections. Section II will provide motivation for the analytical method proposed by Bower and Schultheiss. Section III will introduce the frequency domain design method for minimum-phase continuous-time systems. Section IV will show how the method can 
accommodate time and frequency domain specifications. Section V will expand the method to nonminimum phase and sampled-data systems. Section VI will briefly discuss the undergraduate control theory sequence at Bradley University for electrical and computer engineering students.

\section{Frequency Domain Design}

Most real world systems are better described in the frequency domain. For example, time delay can be treated exactly in the frequency domain. Time domain analysis and design (root locus) is normally the first approach to present in control theory classes. We feel that time domain methods and the associated specifications should be clearly understood before teaching frequency domain approaches. Some textbooks do a better job showing the design procedures and the iterative process than others. A good example is the textbook by Rohrs, Melsa, and Schultz ${ }^{4}$. One design example shown by the authors uses the optimum phase margin method described in the next section.

In undergraduate and introductory graduate textbooks that cover frequency domain design, authors concentrate on shaping the magnitude versus frequency curve using gain, lag, lead, lag-lead, and PIDtype compensators to meet a set of specifications. Little or no attention is given to shaping the phase versus frequency curve. There are a number of textbooks that cover shaping the magnitude curve in detail $^{1,3-8}$. Shaping of the phase curve will be the focus of this paper although the techniques for magnitude shaping must be understood as well.

The open-loop frequency response of a minimum-phase system $\mathrm{G}(\mathrm{s})$ in the vicinity of the crossover frequency $w_{c}$ is shown in Fig. 1. It is always desired to force the magnitude curve's slope at crossover to be -1 (log-log plot) or $-20 \mathrm{db}$ per decade (semi-log plot). This will yield a stable system with a phase margin between 0 and 90 degrees which depends on the length of the -1 segment. The ideal situation for the shape of the phase curve is shown in Fig. 1. The peak of the phase curve occurs at $\mathrm{w}_{\mathrm{c}}$ thus yielding the maximum phase margin and creating a system that is robust to plant variations. A given phase margin (PM) specification will determine the length of the -1 slope segment.

A more important specification to the user after stability is assured is system speed (a time domain specification). Maximizing $\mathrm{w}_{\mathrm{c}}$ will maximize closed-loop bandwidth and speed. Relationships and equations between the time and frequency domains will be covered in Section IV. The objective of the optimum PM margin design method is to shape the phase curve in the form of a peak at $\mathrm{w}_{\mathrm{c}}$ and to increase $w_{c}$ as far as the constraints of the system will allow. Control system design normally requires several design iterations in a simulation environment to meet a set of system specifications. The optimum PM method comprises the first design iteration but normally results in a design that is close to optimum, i.e., the resulting system will have maximum stability margin and bandwidth. 


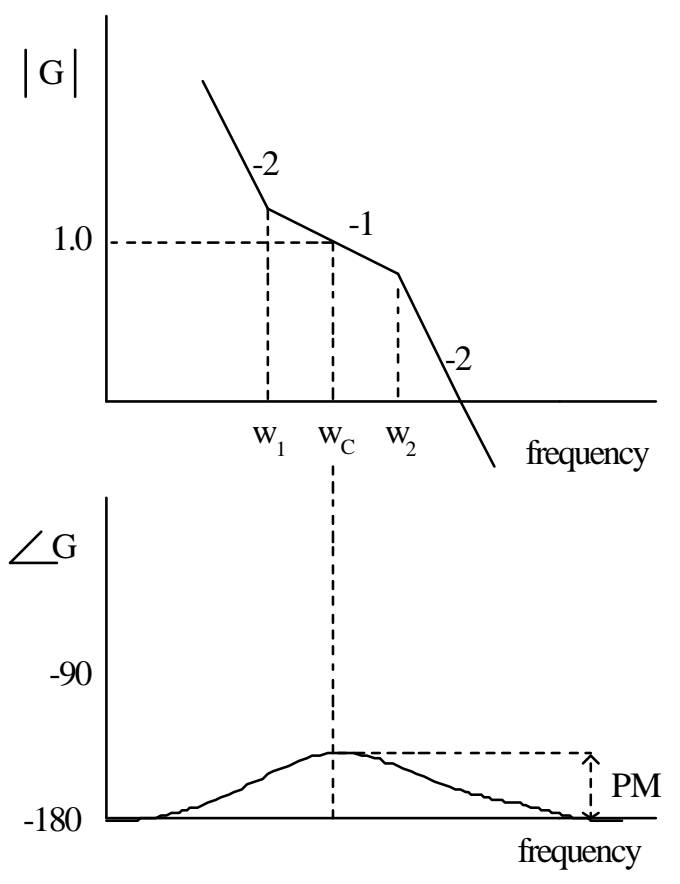

Fig. 1. Open-Loop Frequency Response Example.

\section{Optimum Phase Margin Design}

Phase margin is shown graphically in Fig. 1 or can be stated as

$$
P M=180^{\circ}+\angle G\left(j w_{c}\right)=180^{\circ}+B_{c}
$$

The phase angle $B_{c}$ of the open-loop transfer function $G(s)$ at $w_{c}$ is

$$
B_{c}=-\pi+\tan ^{-1} \frac{w_{c}}{w_{1}}-\tan ^{-1} \frac{w_{c}}{w_{2}}
$$

assuming the -2 segments are infinite length. Equation (2) can be simplified for design purposes as

$$
B_{c} \approx-\pi+\left(\frac{\pi}{2}-\frac{w_{1}}{w_{c}}\right)-\frac{w_{c}}{w_{2}}
$$

using approximations for the arc tan function. Expressing the arc tan function as a power series

$$
\begin{gathered}
\tan ^{-1} x=x-\frac{x^{3}}{3}+\frac{x^{5}}{5}-\cdots \approx x \text { for } \mathrm{x}<1 \\
\tan ^{-1} x=\frac{\pi}{2}-\frac{1}{x}+\frac{1}{3 x^{3}}-\frac{1}{5 x^{5}}+\cdots \approx \frac{\pi}{2}-\frac{1}{x} \text { for } \mathrm{x}>1
\end{gathered}
$$

shows that eliminating the high-order terms introduces minimal error because of the alternating

Proceedings of the 2001 American Society for Engineering Education Annual Conference \& Exposition Copyright @2001, American Society for Engineering Education 


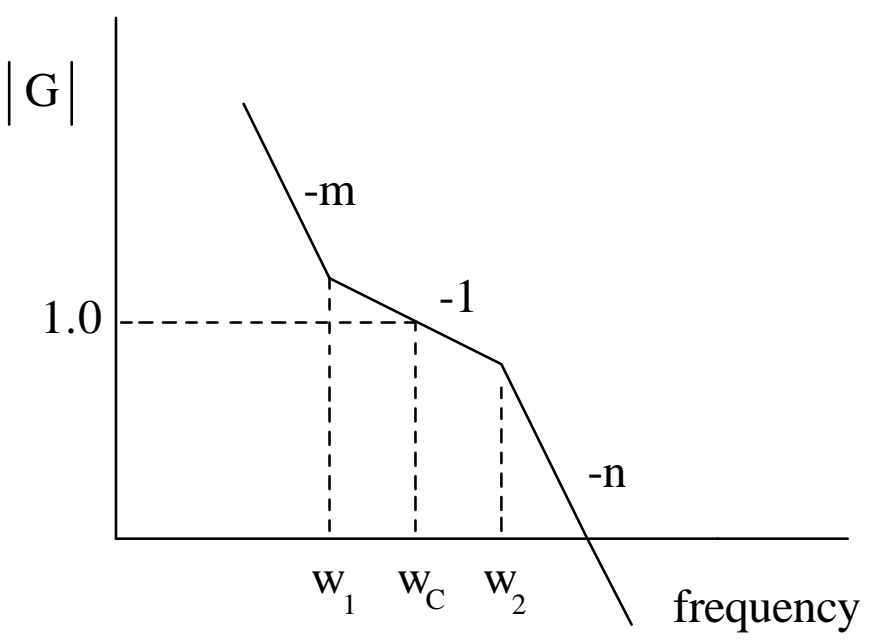

Fig. 2. General Shape in the Vicinity of the Crossover Frequency.

series and the first nonlinear term is cubic. The optimum $w_{c}$ in Fig. 1 can be determined by differentiating (3) with respect to $w_{c}$

$$
\frac{d B_{c}}{d w_{c}}=\frac{w_{1}}{w_{c}^{2}}-\frac{1}{w_{2}}=0 \quad \text { or } \quad w_{c}=\sqrt{w_{1} w_{2}}
$$

Refer to Fig. 2 for derivation of optimum $w_{c}$ for general shapes in the vicinity of $w_{c}$. The phase angle of the open-loop transfer function $\mathrm{G}(\mathrm{s})$ at $\mathrm{w}_{\mathrm{c}}$ is

$$
B_{c}=-\frac{\pi}{2} m+(m-1)\left(\frac{\pi}{2}-\frac{w_{1}}{w_{c}}\right)-(n-1) \frac{w_{c}}{w_{2}}
$$

assuming infinite $\mathrm{m}$ and $\mathrm{n}$ segments. The optimum $\mathrm{w}_{\mathrm{c}}$ for the general shapes is

$$
w_{c}=\sqrt{\frac{m-1}{n-1} w_{1} w_{2}}
$$

Consider the continuous-time system shown in Fig. 3 for an example of applying the optimum PM approach. The plant is

$$
G p(s)=\frac{10}{(0.1 s+1)(0.0005 s+1)}
$$

The system specifications are:

1. steady-state error $\left(\mathrm{e}_{\mathrm{ss}}\right)$ to step inputs $=0$

2. steady-state error $\left(\mathrm{e}_{\mathrm{ss}}\right)$ to ramp inputs $<0.1 \%$

3. phase margin $\geq 50$ degrees

Proceedings of the 2001 American Society for Engineering Education Annual Conference \& Exposition Copyright $\odot 2001$, American Society for Engineering Education 


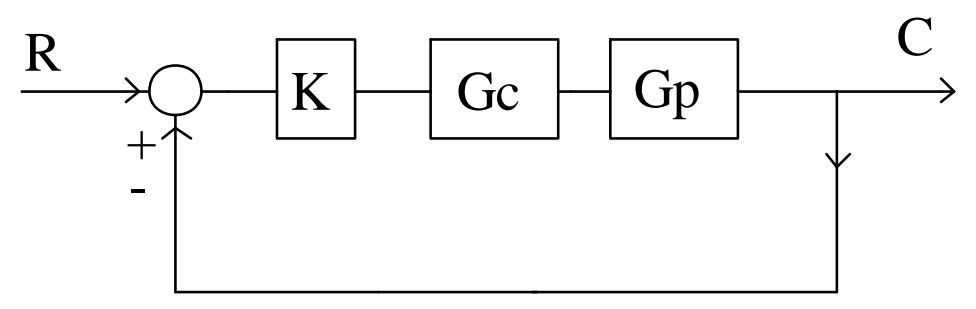

Fig. 3. Continuous-Time Example.

4. minimize rise time $\left(\mathrm{t}_{\mathrm{r}}\right)$ for step inputs

5. noise level (open-loop) $\leq 10 \%$ at frequencies $\geq 1000 \mathrm{rad} / \mathrm{sec}$

The steady-state error specification for step inputs requires the compensator $\mathrm{Gc}(\mathrm{s})$ to have one integration. The ramp error specification is used to determine the proportional gain $\mathrm{K}$ as follows:

$$
e_{s s}=\frac{1}{K_{v}}=0.001 \text { or } K_{v}=1000
$$

where

$$
K_{v}=\lim _{s \rightarrow 0} s K G c(s) G p(s)=10 K=1000
$$

Therefore the proportional gain $\mathrm{K}$ is equal to 100 to just meet the specification. The partially compensated open-loop transfer function is

$$
K G c(s) G p(s)=100\left(\frac{1}{s}\right)\left(\frac{10}{(0.1 s+1)(0.0005 s+1)}\right)
$$

The magnitude versus frequency sketch is shown in Fig. 4 (violet-blue curve). Our students are required to perform the first design iteration on graph paper before using a simulation tool. From Fig. $4, w_{c}$ is 100 $\mathrm{rad} / \mathrm{sec}$. Using the arc tan approximations, the phase angle at $\mathrm{w}_{\mathrm{c}}$ is

$$
B_{c} \approx-\frac{\pi}{2}-\left(\frac{\pi}{2}-\frac{10}{100}\right)-\frac{100}{2000} \approx-3.09 \mathrm{rad} \approx-177^{0}
$$

Using (1), the PM is 3 degrees (almost unstable).

It is desired to crossover with a -1 segment to maximize PM. To minimize rise time (maximize speed in the time domain), a PM of 50 degrees is used. Higher PM results in a slower system (longer rise time). A lead network can produce the desired shape at $\mathrm{w}_{\mathrm{c}}$ and achieve a larger $\mathrm{w}_{\mathrm{c}}$ than the partially compensated system shown in Fig. 4. Refer to Fig. 4 for a quick sketch of the proposed compensated open-loop system (violet-red curve). Note that there are three unknowns, $\mathrm{w}_{1}, \mathrm{w}_{\mathrm{c}}$, and $\mathrm{w}_{2}$, where $\mathrm{w}_{1}$ and $\mathrm{w}_{2}$ will be determined by the lead network. For optimum PM,

Proceedings of the 2001 American Society for Engineering Education Annual Conference \& Exposition Copyright @2001, American Society for Engineering Education 


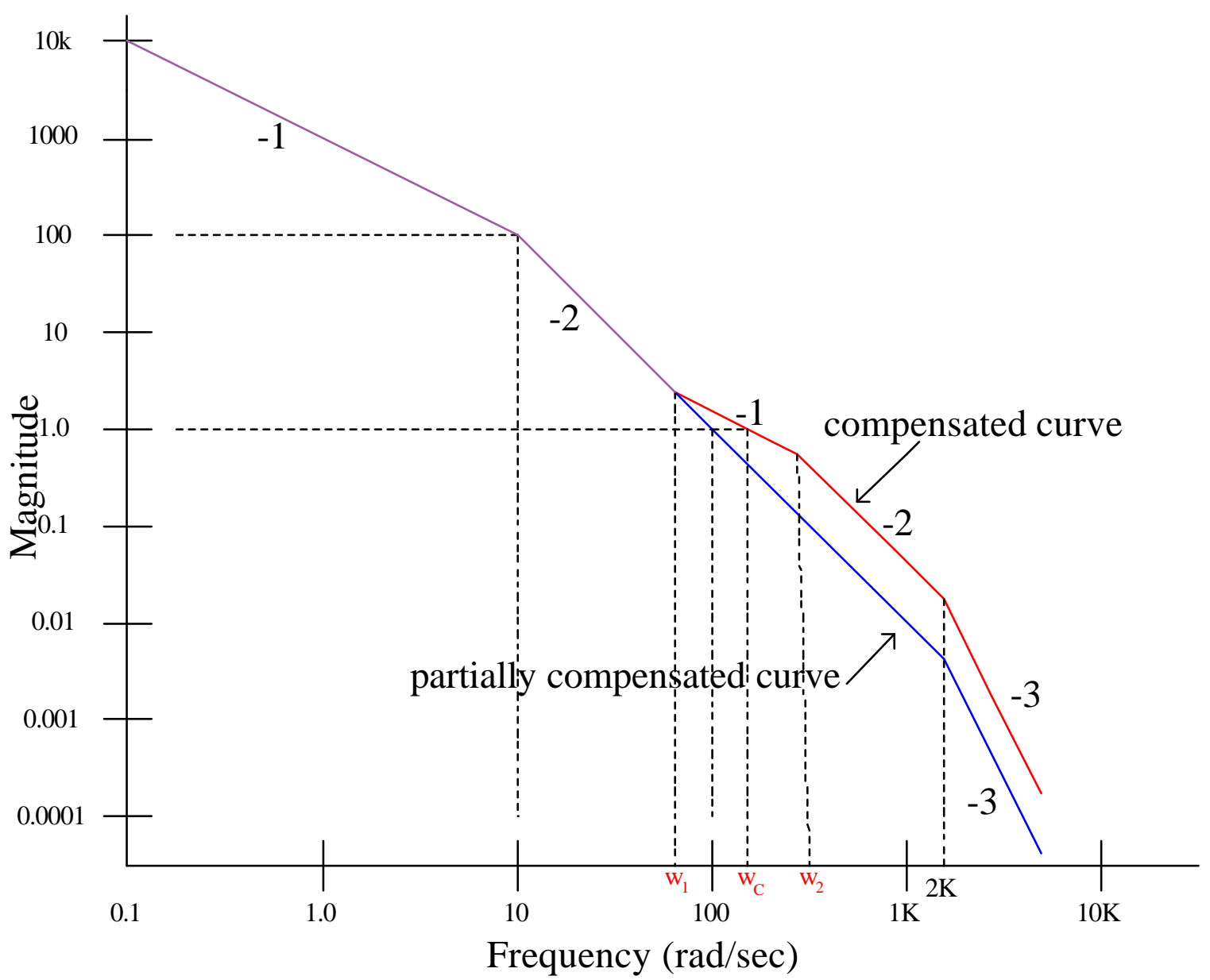

Fig. 4. Quick Sketch of Compensated Open-Loop Magnitude Curve.

$$
w_{c}=\sqrt{w_{1} w_{2}} \text { or } w_{1}=\frac{w_{c}^{2}}{w_{2}}
$$

The desired angle $\mathrm{B}_{\mathrm{c}}$ for a PM of 50 degrees is

$$
B_{c}=-130^{\circ}=-2.27 \mathrm{rad}=-\pi+\left(\frac{\pi}{2}-\frac{w_{1}}{w_{c}}\right)-\frac{w_{c}}{w_{2}}
$$

assuming infinite length segments for the -2 slopes. Substituting $\mathrm{w}_{1}$ from (14), (15) is reduced to

$$
-2.27 \mathrm{rad}=-\frac{\pi}{2}-2 \frac{w_{c}}{w_{2}}
$$

It is desired to maximize $\mathrm{w}_{\mathrm{c}}$ but there are a number of practical constraints. Assuring a reasonable

Proceedings of the 2001 American Society for Engineering Education Annual Conference \& Exposition Copyright $@ 2001$, American Society for Engineering Education 
reduction in noise at high frequencies will limit $\mathrm{w}_{\mathrm{c}}$. Also, the high frequency dynamics of the plant may restrict crossover frequencies. For this example, the noise level specification requires the magnitude of the open-loop transfer function to be $\leq 0.1$ for frequencies $\geq 1000 \mathrm{rad} / \mathrm{sec}$. From a quick sketch of the compensated magnitude curve, $\mathrm{w}_{\mathrm{c}}$ should be selected to be less than $200 \mathrm{rad} / \mathrm{sec}$. For the first design iteration let $\mathrm{w}_{\mathrm{c}}$ equal $150 \mathrm{rad} / \mathrm{sec}$. From (16), $\mathrm{w}_{2}$ is equal to $429 \mathrm{rad} / \mathrm{sec}$ and from (14), $\mathrm{w}_{1}$ is equal to $52 \mathrm{rad} / \mathrm{sec}$. The final first iteration compensator is

$$
K G c(s)=\left(\frac{100}{s}\right) \frac{s / 52+1}{s / 429+1}
$$

This compensator is used to generate the exact magnitude-phase versus frequency curves using the Control System Toolbox from The Math Works Inc. The exact PM is 48.9 degrees with a $\mathrm{w}_{\mathrm{c}}$ of 183 $\mathrm{rad} / \mathrm{sec}$. However, the design is not optimum. The peak of the phase curve is at $110 \mathrm{rad} / \mathrm{sec}$. Reduction of the proportional gain $\mathrm{K}$ from 100 to 54 will align $\mathrm{w}_{\mathrm{c}}$ with the peak of the phase curve. This will yield a PM of 52 degrees.

Unfortunately more design iterations are required because the gain reduction causes the ramp steady-state error to fall out of specification. Experience will lead to minimizing the number of design iterations. A better approach would be to push the peak of the phase curve toward the present crossover frequency. After three more iterations using the Control System Toolbox, the final compensator is

$$
K G c(s)=\left(\frac{100}{s}\right) \frac{s / 65^{+1}}{s / 700^{+1}}
$$

The final compensated open-loop frequency response is shown in Fig. 5. The phase margin is 54 degrees at a crossover frequency of $161 \mathrm{rad} / \mathrm{sec}$. A further increase in $\mathrm{w}_{\mathrm{c}}$ would violate the noise level specification. After the first design iteration is performed on graph paper, we like to refer to subsequent iterations as "educated trial and error" with a simulation package. As stated from the Bower and Schultheiss textbook, "It is not our aim to compile a handbook listing all possible contingencies". We agree with their statement although we have provided numerous continuous-time and sampled-data system examples to our students.

\section{Control Specifications}

The optimum PM approach may require equations that relate time domain or closed-loop frequency domain specifications back to the open-loop parameters PM and crossover frequency. The majority of undergraduate control theory textbooks cover these relationships ${ }^{3-8}$. As an example, one of the more 


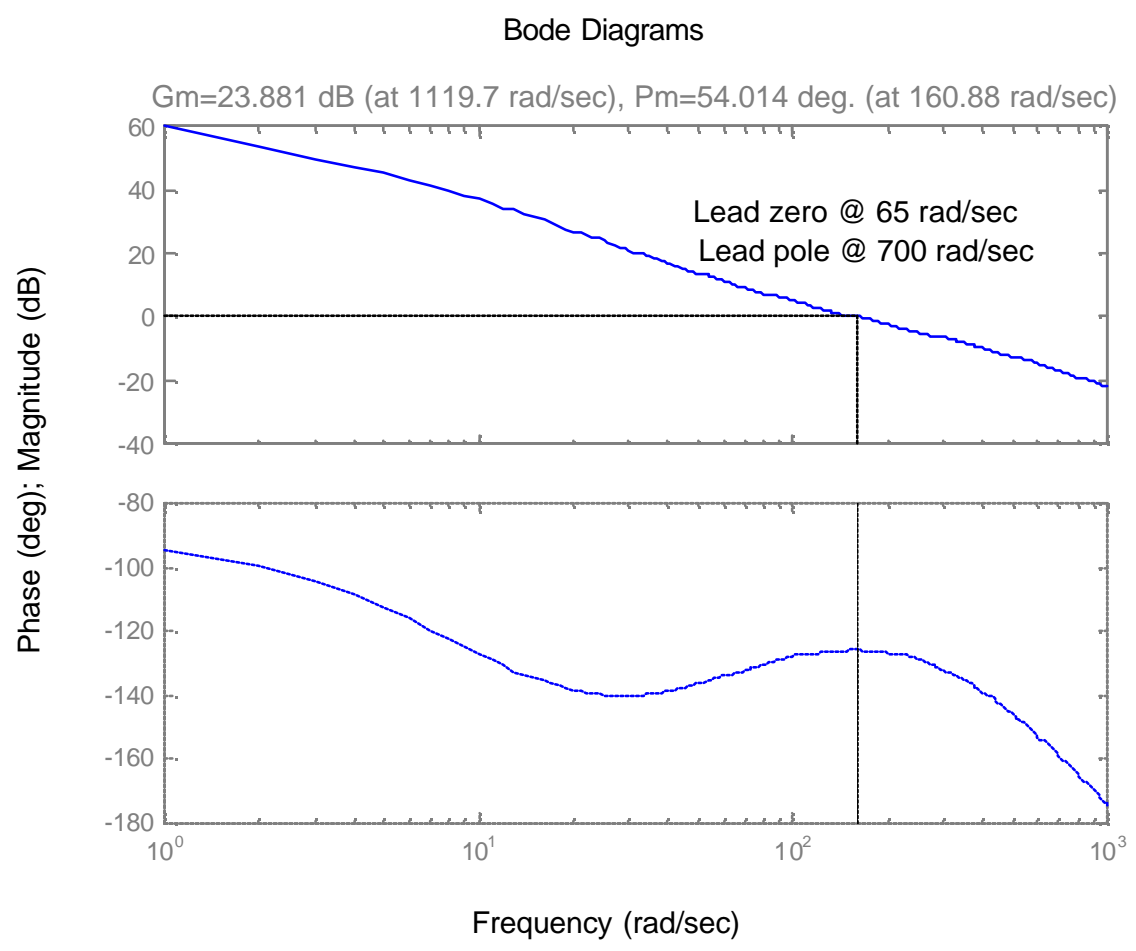

Fig. 5. Final Compensated Open-Loop Frequency Response.

important time domain specifications is percent overshoot for step inputs. In terms of the damping ratio $\delta$, percent overshoot is

$$
\% \text { O.S. }=100 e^{\frac{-\pi \delta}{\sqrt{1-\delta^{2}}}}
$$

An equation to tie this specification back to the open-loop frequency domain is

$$
P M \approx 100 \delta \quad \text { for } P M<70^{\circ}
$$

A critical parameter in the closed-loop frequency domain is $M_{p}$ (maximum peak value). The relationship between $\mathrm{M}_{\mathrm{p}}$ and $\mathrm{PM}$ is

$$
\sin (P M) \approx\left(M_{p}\right)^{-1}
$$

Normally the user can expect more design iterations when the specifications are given in the time domain or closed-loop frequency domain because of the approximation equations. Also the specification equations are derived for an exact second-order system ${ }^{6}$.

\section{Nonminimum-Phase and Sampled-Data Systems}

For minimum-phase systems, phase can be determined indirectly from the magnitude versus frequency

Proceedings of the 2001 American Society for Engineering Education Annual Conference \& Exposition Copyright @2001, American Society for Engineering Education 


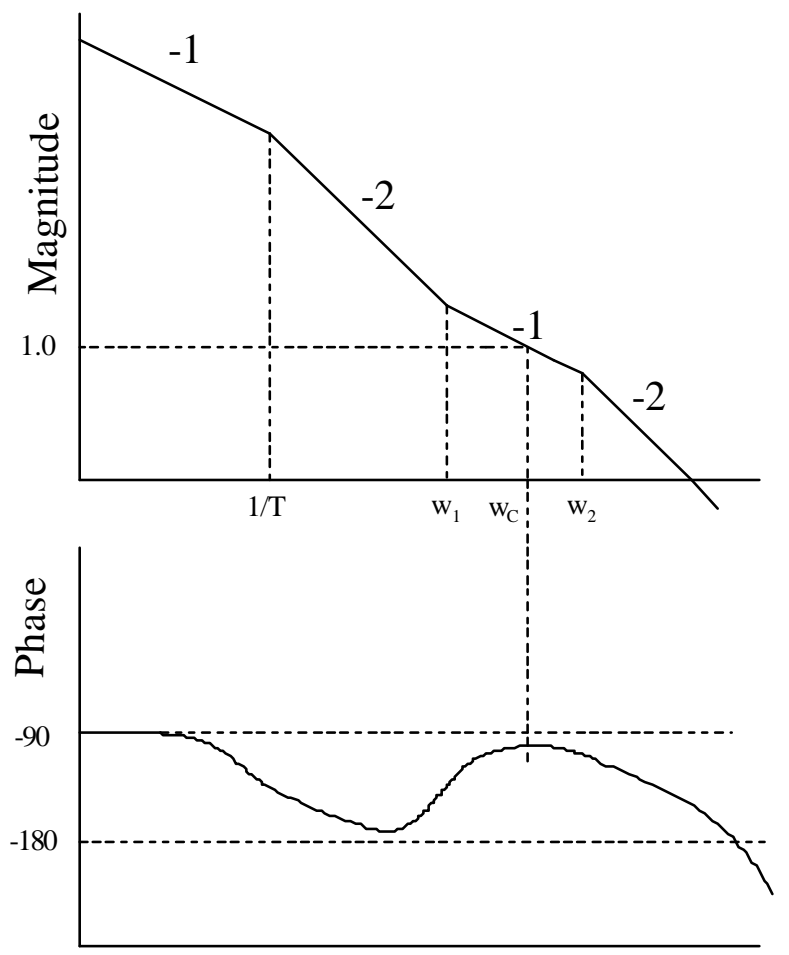

Frequency $(\mathrm{rad} / \mathrm{sec})$

Fig. 6. Nonminimum-Phase System Compensation Example.

curve. Nonminimum-phase and sampled-data systems require using both magnitude and phase versus frequency curves. Consider the system shown in Fig. 3 with a first-order plant with time delay $T_{D}$,

$$
G p=\frac{K e^{-s T_{D}}}{T s+1}
$$

A suggested compensation method is shown in Fig. 6 where

$$
G c=K_{p} \frac{s / z+1}{s(s / p+1)}
$$

The steady-state error and system speed is improved with the PID-type compensation. The angle at crossover is

$$
B_{c} \approx-\pi+\left(\frac{\pi}{2}-\frac{w_{1}}{w_{c}}\right)-\frac{w_{c}}{w_{2}}-w_{c} T_{D}
$$

Differentiating (24) with respect to $\mathrm{w}_{\mathrm{c}}$ and solving for $\mathrm{w}_{\mathrm{c}}$ for the optimum PM yields 


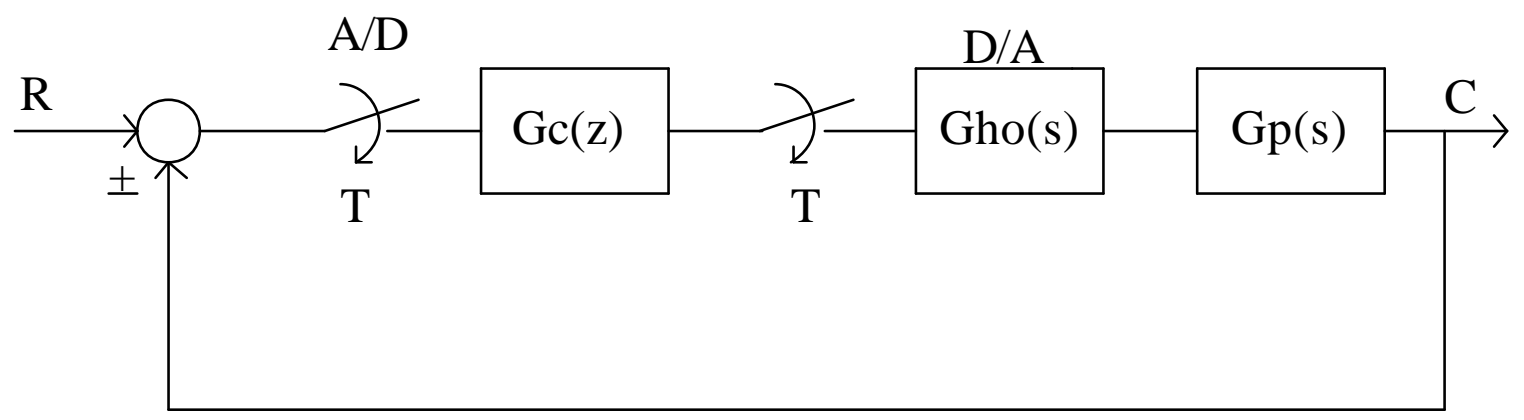

Fig. 7. Sampled-Data System Example.

$$
w_{c}=\sqrt{\frac{w_{1}}{T_{D}+1 / w_{2}}}
$$

The suggested compensation method in Fig. 6 may not be practical for systems with a large time delay component at $\mathrm{w}_{\mathrm{c}}$. The lead portion of the compensator is used to cancel the effect of the time delay's phase lag at $\mathrm{w}_{\mathrm{c}}$. However, large time delays may require a long -1 segment which may not be feasible for some systems.

Consider the sampled-data system shown in Fig. 7. In this application, the compensation is implemented in software (Gc(z)). A digital-to-analog converter (Gho) is used to drive the continuous-time plant $\mathrm{Gp}(\mathrm{s})$. The $\mathrm{A} / \mathrm{D}$ converter is modeled as an ideal sampler.

Method 1: A continuous-time approach can be used to determine Gc(s) using an approximate model for the zero-order hold device. Once $\mathrm{Gc}(\mathrm{s})$ is obtained, $\mathrm{Gc}(\mathrm{z})$ can be found using one of the common discretization methods such as the bilinear transformation?. In the frequency domain, the zero-order hold is described as

$$
G h o(j w)=T \frac{\sin \left(\pi w / w_{s}\right)}{\pi w / w_{s}} \text { with angle }=-\pi w / w_{s}
$$

where $\mathrm{w}_{\mathrm{s}}$ is the sampling frequency. For a sampling rate $\mathrm{w}_{\mathrm{s}} \geq 6 \mathrm{w}_{\mathrm{c}}$,

$$
\text { Gho } \approx 1.0 \text { with an angle }=-\pi w / w_{s}
$$

The $B_{c}$ equation for the system requires a simple modification of adding the phase lag term for the

Proceedings of the 2001 American Society for Engineering Education Annual Conference \& Exposition Copyright $\odot 2001$, American Society for Engineering Education 
zero-order hold. The design procedure is similar to the method discussed previously for a system with time delay.

Method 2: Design in the w-plane is an alternative method for determining Gc(z) in Fig. 7. The exact representation of the zero-order hold is used in this method. A right-half plane zero is produced in the w-plane due to the zero-order hold. The design method is discussed in Ogata's textbook ${ }^{9}$. The optimum PM design in the w-plane is similar to the methods shown previously. Once $\mathrm{Gc}(\mathrm{w})$ is determined, the inverse transformation is used to describe $\mathrm{Gc}(\mathrm{z})$.

\section{Undergraduate Control Sequence at Bradley University and Concluding Remarks}

The first control theory course is offered fall semester of the senior year. This classical control course is divided into three equal sections: modeling, time domain analysis and design (root locus), and frequency domain analysis and design. Gain, lag, lead, lag-lead, and PID-type controllers are introduced with the root locus design method. Only gain compensation is discussed with the frequency domain approach because of time constraints but the type of plants are open-ended including plants with time delay.

The second control theory course (spring semester) is divided into three sections: continuation of frequency domain design (20\%), digital control (40\%), and the state-variable control method (40\%). The course begins with frequency domain compensation (shaping) to meet a set of specifications for continuous-time systems. Gain, lag, lead, lag-lead, and PID-type controllers are introduced as well as the optimum PM approach. Frequency domain and optimum PM are again the primary design methods for the digital control section of the course. The last $40 \%$ of the course introduces state-variable modeling, analysis, and design. Note that the emphasis in the two course sequence is classical control. The first graduate control course is devoted to the state-variable method.

In the undergraduate classes, the optimum frequency domain design methods are applied to several types of analog systems including ones with time delay and also sampled-data systems. Students are required to perform anywhere from 2 to 5 design iterations in homework and take-home exam problems. Because of the different systems (electronic, thermal, hydraulic, mechanical) and types of signals (analog, digital, or mixture), new equations must be derived to account for these differences. These equations are applied in the first design step. The next design step involves use of control system simulation software. The Control System Toolbox, MATLAB, and SIMULINK has been the platform used at Bradley. Once the control system is optimized in simulation, the controller is then converted to software or hardware for implementation.

The optimum phase margin approach has been used in senior capstone projects which have consisted of phase-locked loops, switching power supplies, and a temperature controller. It is currently being applied to a small robot arm system by two senior students and to an industrial hydraulic application by a

Proceedings of the 2001 American Society for Engineering Education Annual Conference \& Exposition Copyright (C2001, American Society for Engineering Education 
graduate student for her thesis. Although the method requires a mathematical description of the plant, a high precision model is not required. It is especially useful in electronic control applications where the model can usually be determined directly from a data sheet. Examples are switching power supply integrated circuits and phase-locked loop circuits.

\section{Bibliography}

1. Bower, J. L. and Schultheiss, P. M. Introduction to the Design of Servomechanisms. John Wiley \& Sons, (1958).

2. Eveleigh, V.W. Adaptive Control and Optimization Techniques. McGraw-Hill Book Company (1967).

3. Eveleigh, V.W. Introduction to Control Systems Design. McGraw-Hill Book Company (1972).

4. Rohrs, C. E., Melsa, J. L., and Schultz, D. G. Linear Control Systems. McGraw-Hill, Inc. (1993).

5. D'Azzo, J. J. and Houpis, C. H. Linear Control System Analysis and Design. McGraw-Hill, Inc. (1995).

6. Franklin, G. F., Powell, J. D., and Emami-Naeini, A. Feedback Control of Dynamic Systems. Addison-Wesley Publishing Company (1991).

7. Kuo, B. C. Automatic Control Systems. Prentice Hall (1991).

8. Ogata, K. Modern Control Engineering. Prentice Hall (1997).

9. Ogata, K. Discrete-Time Control Systems. Prentice Hall (1987).

\section{GARY L. DEMPSEY}

Gary Dempsey received the Ph.D. in Electrical Engineering from the University of Virginia in 1991. Dr. Eugene McVey served as dissertation advisor. He is currently an Associate Professor in the Electrical and Computer Engineering Department at Bradley University in Peoria, IL. He currently teaches the undergraduate control theory sequence, senior and graduate laboratories, and artificial neural networks.

\section{EUGENE S. McVEY}

Eugene McVey received the Ph.D. degree in engineering from Purdue University in 1960. He was Instructor and Assistant Professor from 1957 to 1961 at Purdue University. From 1962 to 1994, he was Associate Professor (19611966) and Professor (1966-1994) at the University of Virginia. He is the author of over 140 archival publications, holds 18 patents, and supervised $30 \mathrm{Ph} . \mathrm{D}$. dissertations and 55 Master Theses during his teaching career. 